\title{
Numerical and experimental study on the impact of chromatic dispersion on O-band direct-detection transmission
}

\author{
Yang Hong*, Kyle R. H. Bottrill, Natsupa Taengnol, Naresh K. \\ ThIPPARAPU, Yu WANG, JAYANTA K. SAHU, DAVID J. RICHARDSON, AND \\ PERIKLIS PETROPOULOS \\ Optoelectronics Research Centre, University of Southampton, Southampton SO17 1BJ, United Kingdom \\ *y.hong@soton.ac.uk
}

\begin{abstract}
The recent emergence of efficient O-band amplification technologies has enabled the consideration of O-band transmission beyond short-reach. Despite the O-band being a low chromatic dispersion $(\mathrm{CD})$ window, the impact of $\mathrm{CD}$ will become increasingly significant when extending the reach of direct-detection (DD) systems. In this work, we first numerically investigate the 3-dB bandwidth of single-mode fibers (SMF) and the CD-restricted transmission reach in intensity-modulation DD systems, confirming the significant difference between lowand high-dispersion O-band wavelengths. We then carry out experimental transmission studies over SMF for distances of up to $70 \mathrm{~km}$ at two different wavelengths, the low-dispersion $1320 \mathrm{~nm}$ and the more dispersive $1360 \mathrm{~nm}$, enabled by the use of an O-band bismuth-doped fiber amplifier as a pre-amplifier at the receiver. We compare three 50-Gb/s optical DD formats, namely Nyquist on-off keying (OOK), Nyquist 4-ary pulse amplitude modulation (PAM4) and Kramers-Kronig detection-assisted single-sideband quadrature phase shift keying (KK-QPSK) half-cycle subcarrier modulation. Our results show that at both wavelengths, OOK and QPSK exhibit better bit error rate performance than PAM4. When transmitting over 70-km of SMF at the less dispersive wavelength of $1320 \mathrm{~nm}, 50-\mathrm{Gb} / \mathrm{s}$ OOK modulation offers more than $1.5-\mathrm{dB}$ optical power sensitivity improvement at the photodiode (PD) compared to 50-Gb/s QPSK. Conversely, at $1360 \mathrm{~nm}$, the required optical power to the PD can be reduced by more than 3 $\mathrm{dB}$ by using QPSK instead of OOK.
\end{abstract}

(C) 2021 Optical Society of America under the terms of the Creative Commons Attribution 4.0 License.

\section{Introduction}

Optical data transmission in the O-band of the spectrum $(1260-1360 \mathrm{~nm})$ retains its relevance in short-reach $(<40 \mathrm{~km})$ applications, benefitting from both the low chromatic dispersion in standard silica single-mode fibers (SMFs) and a mature and low-cost transceiver technology [1-3], which together allow for high-speed communications using simple intensity modulation and direct-detection (IM/DD) [4]. Various efforts have been reported in the literature in this spectral band, including novel transceiver designs and high-speed demonstrations involving both a single and multiple wavelengths [5-7].

The use of the O-band in transmission systems is generally restricted to short-haul applications, mainly because of: (i) the relatively higher fiber loss (compared to the C-band), indeed this loss difference becomes more significant with an increase in the transmission distance, and (ii) the lack of efficient optical amplification in this wavelength range [8]. In contrast, the C-band, apart from enjoying lower fiber propagation losses, also benefits from the availability of erbium-doped fiber amplifiers, making it preferable when considering longer reach applications. In this regard, further reach extension of O-band systems can only be made possible once satisfactory amplification solutions are available. Optical amplification in the Oband has typically relied on the use of either semiconductor optical amplifiers (SOAs) or Praseodymium-doped fiber amplifiers (PDFAs) [9]. However, the SOA suffers from a high 
noise figure, nonlinear distortions and normally exhibits a high polarization sensitivity, whilst the PDFA offers a relatively narrow gain bandwidth and is costly, since it is not based on a silica host glass [9-10]. Recently, the emergence of diode-pumped bismuth-doped fiber amplifiers (BDFAs) has offered hope for a more promising solution for efficient O-band amplification [9-13]. A few initial studies based on the use of BDFAs have already been demonstrated, confirming their advantages over SOAs, such as much lower nonlinear distortions, higher gain and wider bandwidth [9, 13-15]. Furthermore, the feasibility of highspeed transmission using the BDFA for O-band amplification [16-17], including a 320-Gb/s DD transmission experiment over a 50-km length of SMF was recently reported [17]. Therefore, we anticipate that the reach of currently deployed coarse wavelength division multiplexed (CWDM) O-band systems can be extended through the use of BDFAs.

On the other hand, most of the previous transmission demonstrations focused on wavelengths within a narrow near-zero-dispersion window to minimize the impact of chromatic dispersion (CD) [5-7]. Considering the gain bandwidth of the aforementioned O-band BDFAs, it is possible to realize high-speed transmission over longer distances across the O-band. However, the impact of CD can no longer be neglected when extending the reach of O-band systems and adopting more dispersive wavelengths. This results from the fact that the $\mathrm{CD}$ in SMF is cumulative, thus despite the O-band being a low chromatic dispersion (CD) window, the accumulating effects of CD may still cause severe power fading in IM/DD transmission, and thus limit the transmission performance [17-18]. This becomes more prominent and can be a critical issue when pushing the currently standardized short-reach $50-\mathrm{Gb} / \mathrm{s} / \lambda \mathrm{CWDM} \mathrm{O}-$ band systems (e.g. specified in IEEE 802.3bs [19]) to longer-reach scenarios, e.g. inter data center interconnects, as enabled by novel O-band fiber amplifier technologies. To this end, the investigation of modulation formats that are commonly specified in relevant standards, including non-return-to-zero on-off keying (NRZ-OOK) and 4-ary pulse amplitude modulation (PAM4), is critical for longer-reach O-band transmission.

In this work, we present both numerical and experimental studies of the impact of $\mathrm{CD}$ on O-band transmission. In our numerical simulations, we chose a signal wavelength of $1320 \mathrm{~nm}$ to investigate the performance near the zero-dispersion wavelength of the SMF, whilst 1360 $\mathrm{nm}$ was used to represent a more dispersive O-band wavelength. We contrast the impact of CDinduced power fading at the two wavelengths after transmission over 70-km of SMF. Consequently, we show that the achievable transmission reach at 1320-nm is around 5.4 times longer than that at $1360-\mathrm{nm}$. We then experimentally implemented a DD transmission system using these two wavelengths, to evaluate the transmission performance of Nyquist NRZ-OOK and Nyquist PAM4 over varying distances, under the respective impact of CD. Kramers-Kronig (KK) detection-assisted single-sideband quadrature phase shift keying half-cycle subcarrier modulation (QPSK-SCM) [20] was also included in the study since it offers significantly enhanced tolerance to CD-induced power fading. We focused our study on $50-\mathrm{Gb} / \mathrm{s}$ DD signals (i.e., 50 GBaud for OOK, and 25 GBaud for PAM4 and QPSK-SCM), which is the currently deployed data rate per wavelength in short-reach O-band systems, and gradually extended the reach up to $70 \mathrm{~km}$ with the aid of a BDFA as a pre-amplifier. Our experiments show that at the near zero-dispersion 1320-nm wavelength, OOK always exhibits the optimal bit error rate (BER) performance at all reaches ranging from $10 \mathrm{~km}$ to $70 \mathrm{~km}$. In comparison, at the more dispersive 1360-nm channel, KK-QPSK shows better BER performance at distances beyond $50 \mathrm{~km}$. At this wavelength and over a distance of $70 \mathrm{~km}$, more than $3-\mathrm{dB}$ receiver sensitivity improvement was achieved at a BER threshold of $3.8 \times 10^{-3}$, i.e., the $7 \%$ forward error correction (FEC) limit, when using the KK-QPSK format. To the best of our knowledge, this work constitutes the first comparative investigation on the performance of $50-\mathrm{Gb} / \mathrm{s}$ double-sideband and single-sideband formats in the O-band at distances that extend to $70 \mathrm{~km}$.

\section{Numerical simulations}


In this Section, we numerically study the impact of CD on O-band IM/DD systems. The values of the $\mathrm{CD}$ used at different wavelengths across the O-band are shown in Fig. 1(a), wherein the corresponding range of CD values specified in the ITU G.652 standard is also included. The smallest and largest $C D$ values within this spectral range were assumed to be around -5.1 and $3.9 \mathrm{ps} / \mathrm{nm} / \mathrm{km}$ at $1260 \mathrm{~nm}$ and $1360 \mathrm{~nm}$, respectively. Using these CD values, we have simulated the corresponding 3-dB bandwidths of the SMF for IM/DD transmission after $10 \mathrm{~km}$ and 70 $\mathrm{km}$, respectively, as shown in Fig. 1(b). Note that the 3-dB bandwidth of SMF here refers to the frequency at which the signal power is reduced by $3 \mathrm{~dB}$ when considering the $\mathrm{CD}$-induced power fading. It is seen that after $10-\mathrm{km}$, a bandwidth broader than $30 \mathrm{GHz}$ can be used for transmission across the O-band. In comparison, after transmission over a 70-km length of SMF, the 3-dB bandwidth is reduced to just $\sim 10 \mathrm{GHz}$ at wavelengths on the edge of the band. In both cases, as expected, the wavelengths close to the zero-dispersion wavelength of the SMF of $\sim 1310 \mathrm{~nm}$ exhibit the broadest bandwidths.
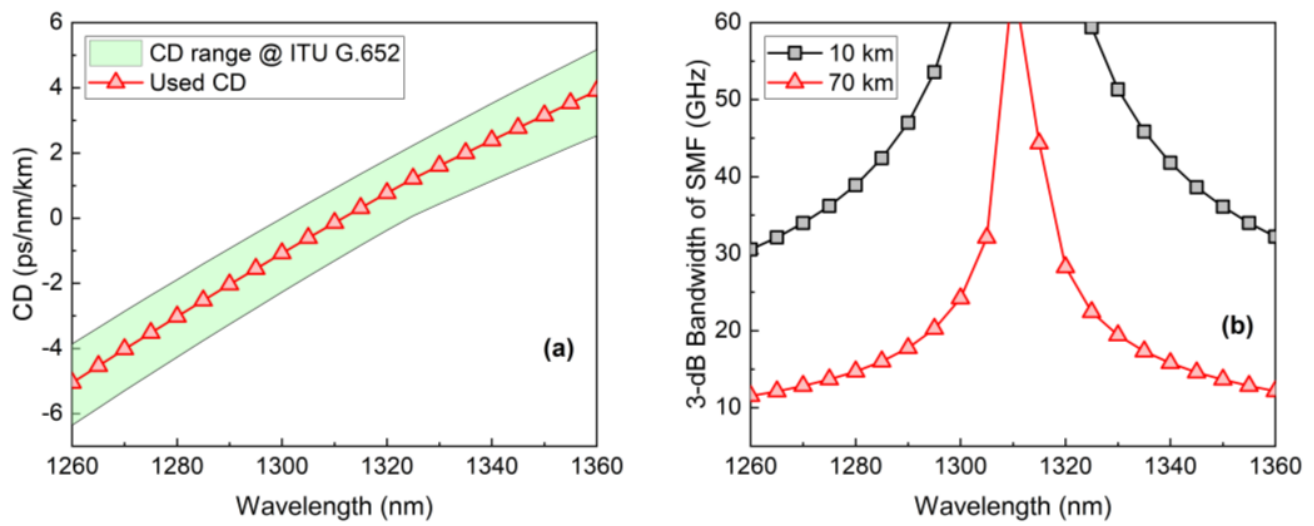

Fig. 1. (a) CD values of SMF in the O-band, and (b) numerical results of 3-dB bandwidth of SMF for IM/DD transmission.

We further used $1320 \mathrm{~nm}$ and $1360 \mathrm{~nm}$ as examples of a near-zero and a more dispersive wavelength to investigate the impact of power fading after 70-km length of SMF and the allowable transmission reaches for different signal bandwidths, and the results are shown in Fig. 2. The normalized response in Fig. 2(a) is the power spectrum of the signal after IM/DD transmission in the SMF. Here, the CD values of the two wavelengths were assumed to be $\sim 0.8$ and $3.9 \mathrm{ps} / \mathrm{nm} / \mathrm{km}$, respectively, which agree well with what is observed in the transmission experiments that follow in Section 4.2. As shown in Fig. 2(a), when using the 1320-nm wavelength, the CD-induced power fading is minor for frequencies up to $25 \mathrm{GHz}$ after $70-\mathrm{km}$ transmission. In contrast, a spectral null occurs at around $17.5 \mathrm{GHz}$ if transmitting at $1360 \mathrm{~nm}$ instead. The spectral null results from the CD-induced phase difference between the optical carrier and the sidebands of the signal, which leads to a succession of nulls in the power spectrum, an effect commonly referred to as power fading in IM/DD transmission [4]. As a result, the 1320-nm wavelength is capable of supporting $~ 5.4$ times longer reach than that of the 1360-nm wavelength, as shown in Fig. 2(b). Note that the reach here is defined as the transmission distance after which the full bandwidth of the baseband electrical signal (i.e., the $x$-axis in Fig. 2(b)) will experience 3-dB power fading. More details regarding this definition can be found in [21]. It is worth noting that the CD effects on DD transmission discussed above can be combated/compensated for in different ways. One viable solution is to use dispersion compensating fibers in the transmission link, which however will introduce additional link loss as well as nonlinearity issues. On the other hand, advanced signal processing [22] and the single-sideband modulation (e.g., the KK-QPSK used in this work) can also be adopted to combat the CD effect. We note that the impact of higher-order dispersion is negligible for the reaches discussed in this work [23], hence is not considered here. 

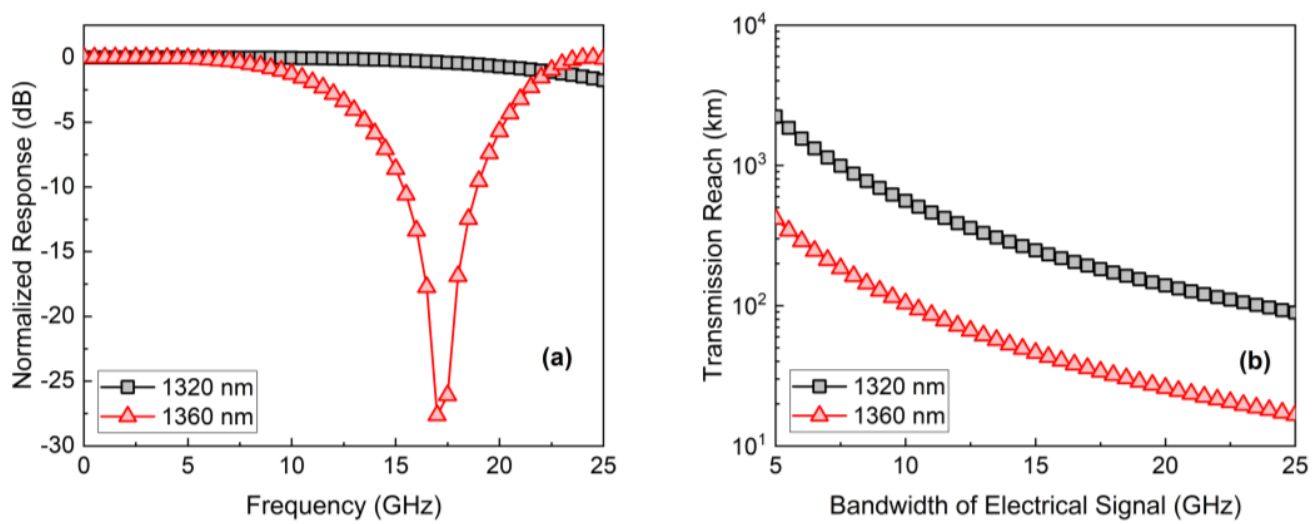

Fig. 2. (a) The profiles of CD-induced power fading after a 70-km length of SMF using the 1320-nm and 1360-nm wavelengths, and (b) allowable transmission reach versus bandwidth of the electrical signal for $1320 \mathrm{~nm}$ and $1360 \mathrm{~nm}$..

\section{Experimental setup}

To evaluate the impact of CD-induced power fading on the transmission performance of different modulation formats, we experimentally implemented a 50-Gb/s O-band DD system using the same two wavelengths of $1320 \mathrm{~nm}$ and $1360 \mathrm{~nm}$. Figure 3 shows the experimental setup, as well as the corresponding block diagrams of the offline DSP used. At the transmitter, an O-band tunable laser operating at $1320 \mathrm{~nm}$ or $1360 \mathrm{~nm}$ was used as the optical carrier. The output power of the tunable laser was set at $13 \mathrm{dBm}$ for both wavelengths. A dual-drive MachZehnder modulator (DD-MZM) was used to generate the OOK/PAM4/QPSK-SCM signals. This was realized by using two individual channels of an arbitrary waveform generator, followed by respective electrical amplifiers (EAs). Note that the DD-MZM was biased at the quadrature point $(3.5 \mathrm{~V})$ for all of the three modulation formats and the driving signal amplitudes for different formats were optimized separately to ensure a fair comparison. The output of the DD-MZM was then launched into 10/20/35/50/70-km lengths of SMF, and the launch power to the SMF was around $3 \mathrm{dBm}$. For the $10-\mathrm{km}$ and $20-\mathrm{km}$ cases, no optical amplification/filtering was adopted, whilst a BDFA [12] and a 1.2-nm optical bandpass filter (OBPF) were used in the $35 / 50 / 70-\mathrm{km}$ cases for pre-amplification and out-of-band noise filtering at the receiver, respectively. The corresponding losses of the SMFs were measured to be around 3.2/6.4/11.8/17.1/24.2 dB, respectively. Therefore, for the 35/50/70-km cases, the input power to the BDFA was around -8.8/-14.1/-21.2 dBm, respectively. The insertion loss of the OBPF was less than $1.5 \mathrm{~dB}$. Subsequently, a variable optical attenuator was used to control the optical power that was injected into the photodetector (PD, Finisar XPRV2022A) which has an integrated trans-impedance amplifier. A 99:1 tap coupler was used to monitor the optical power to the PD. The detected signal at the PD was captured by an $80-\mathrm{GSa} / \mathrm{s}$ digital storage oscilloscope (DSO) for further offline processing. We note that in this length-resolved study, the BDFA was not employed when the transmission distance was not long enough $(<35 \mathrm{~km})$ to justify its use. Therefore, for consistency, whenever the BDFA was in place (transmission over $35 / 50 / 70 \mathrm{~km}$ ), it was considered to be part of the transmission line, and we therefore varied the power fed into the PD whenever we needed to evaluate the BER and signal-to-noise ratio (SNR) performance of the different formats. This methodology is appropriate in this study since our scope is to study the impact of $\mathrm{CD}$ on the data transmission. 

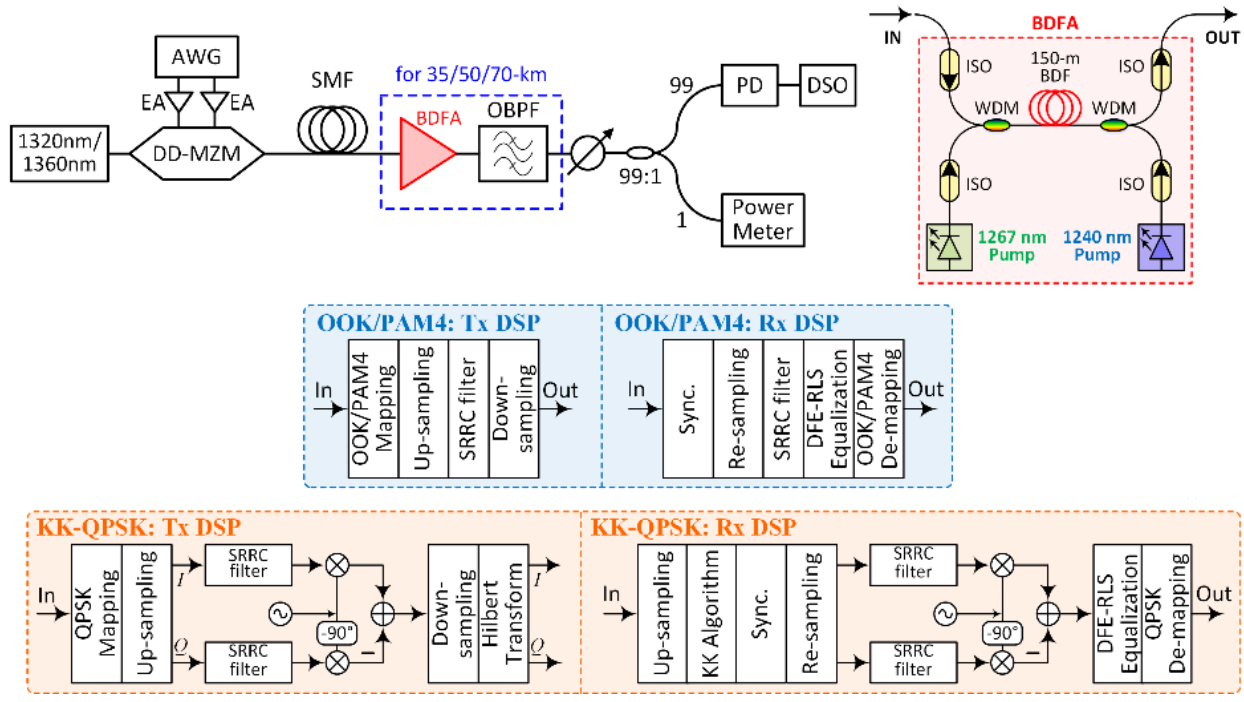

Fig. 3. Experimental setup and the configuration of the BDFA (upper), and the block diagrams of the offline DSP of PAM4/OOK and KK-QPSK (lower).

A sketch of the BDFA is also depicted in Fig. 3. It includes a 150-m length of phosphosilicate bismuth-doped fiber, which was pumped bidirectionally by two laser diodes operating at $1267 \mathrm{~nm}$ and $1240 \mathrm{~nm}$, respectively, to ensure $>40-\mathrm{nm}$ broadband operation. The total power of the two lasers is around $720 \mathrm{~mW}$. For both the $1320-\mathrm{nm}$ and $1360-\mathrm{nm}$ wavelengths used in this work, the BDFA offers a gain of around $22 \mathrm{~dB}$ whilst the noise figure at $1360 \mathrm{~nm}$ was slightly smaller than at $1320 \mathrm{~nm}[12,24]$, ensuring that the BDFA had a negligible impact on comparing the performance of 1320-nm and 1360-nm transmission in this work. Note that while we focused on single-wavelength operation in this work, the BDFA with its $>40-\mathrm{nm}$ bandwidth and $>20-\mathrm{dB}$ gain, is also suitable for use as a booster/in-line/preamplifier in CWDM and dense WDM applications, as has been validated in [14-16, 25].

The signals were generated and prepared for transmission offline. In the cases of OOK/PAM4, the binary inputs were first mapped into OOK/PAM4 symbols. The mapped symbols were then 90-times up-sampled before Nyquist shaping was applied via the use of a square-root-raised-cosine filter (SRRC). The roll-off factor of the SRRC filter was 0.1. The filtered signal was then 50-times (OOK) or 25-times (PAM4) down-sampled. The resulting sequence and its inverse were fed into two individual channels of the AWG operating at 90 $\mathrm{GSa} / \mathrm{s}$, and the generated complementary signals were finally used to drive the DD-MZM. Upon reception, the captured signal from the DSO was first synchronized and then re-sampled before performing the matched SRRC filtering. After that, a half-symbol-spaced decision feedback equalizer (DFE) with 17 feedforward taps and 7 feedback taps was implemented, wherein the recursive least-squares (RLS) algorithm with a forgetting factor of 0.998 was used. The equalized signal was finally OOK/PAM4 de-mapped, and the corresponding BER was obtained via error counting by comparing the de-mapped bits with the original input bits. For the KKQPSK, QPSK mapping was performed before 90-times up-sampling was carried out. A pair of SRRC filters with a roll-off factor of 0.1 (the same as in the OOK/PAM4 cases) were then used to realize Nyquist shaping for the I and Q components of the up-sampled signal. Following this, the obtained I and Q signals were up-converted to a subcarrier frequency of 0.52 times the symbol rate, and then subtracted from one another before 25-times down-sampling. Finally, a digital Hilbert transform was performed and the resulting I and Q signals were sent to the two channels of the 90-GSa/s AWG. At the receiver, the captured signal was first 6-times upsampled before using the KK algorithm [26] to recover the I and Q components. The resulting signal went through synchronization, re-sampling, SRRC matched filtering, down-conversion 
and the same DFE-RLS equalization as used in the OOK/PAM4 cases, and finally QSPK demapping was performed. We highlight that to make a fair comparison, the DSP was nearly identical in all of the three modulation formats, apart from an additional up-sampling and KK detection that were implemented for the recovery of QPSK-SCM. As for the device requirements, the experimental setup also remained unchanged for all formats to ensure fairness in the investigations.

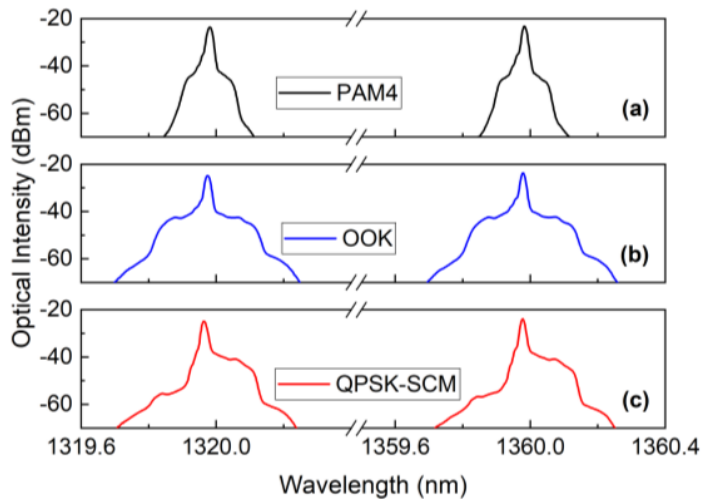

Fig. 4. Optical spectra of the 50-Gb/s signals: (a) PAM4, (b) OOK, and (c) QPSK-SCM. The resolution was $0.01 \mathrm{~nm}$ for all cases.

Figure 4 shows the corresponding optical spectra of the 50-Gb/s PAM4, OOK and QPSKSCM signals in the 1320-nm and 1360-nm cases. It is seen that the OOK signal occupied twice the bandwidth compared to PAM4, as expected. For the QPSK-SCM signals, the shortwavelength sidebands were suppressed with a suppression ratio of around $20 \mathrm{~dB}$, and their optical spectral efficiency is comparable to that of PAM4.

\section{Experimental results}

\subsection{BER and SNR versus optical power to $P D$}

We first present BER and SNR results that were obtained at the two wavelengths of $1320 \mathrm{~nm}$ and $1360 \mathrm{~nm}$ over three lengths of SSMF, namely 10, 35 and $70 \mathrm{~km}$. The BERs versus optical powers to the PD and the corresponding SNRs after 10-km of SMF are shown in Fig. 5. As shown in Figs. 5(a)\&(c), the best receiver sensitivity was achieved when using the OOK format. Although PAM4 occupies around half the electrical bandwidth of both the OOK and the QPSK signals (hence has a greater tolerance to $\mathrm{CD}$ ), it requires a substantially higher linearity and SNR to achieve the same BER as either OOK or QPSK. Therefore, it exhibited the greatest power penalty at both wavelengths.
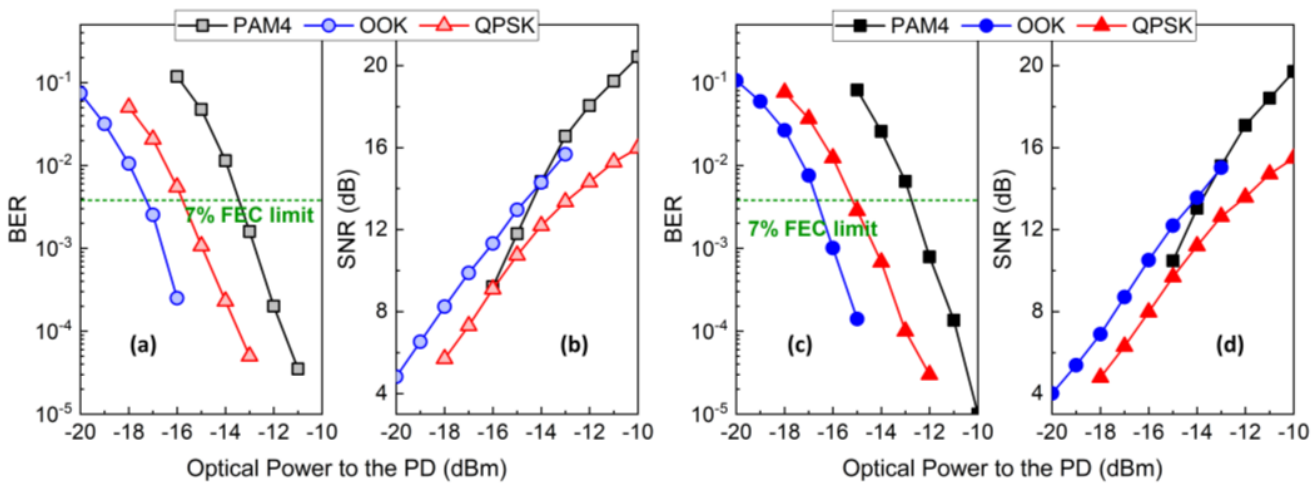
Fig. 5. The transmission performance after a 10-km length of SMF at $1320 \mathrm{~nm}$ : (a) BER versus optical power to the PD and (b) SNR versus optical power to the PD; and at $1360 \mathrm{~nm}$ : (c) BER versus optical power to the PD, and (d) SNR versus optical power to the PD.

At both wavelengths, OOK offered receiver sensitivity improvements of $\sim 1.5 \mathrm{~dB}$ and $\sim 4$ $\mathrm{dB}$ compared to QPSK and PAM4 at the 7\% FEC limit, respectively. At the same optical power to the PD, OOK showed a SNR advantage of $\sim 2.5 \mathrm{~dB}$ over QPSK, as shown in Fig. 5(b)\&(d). This was mainly attributed to the inferior receiver sensitivity of KK detection compared to IM/DD [27-28]. The non-ideal method for generating the single-sideband signal generation using the DD-MZM has also contributed to this SNR penalty. Finally, the figure indicates that for each individual format, the BER and SNR performance were comparable at the two wavelengths. This results from the fact that at this relatively short transmission distance, the impact of CD effects is minimal, as has been indicated in Fig. 1(b).

The corresponding transmission results obtained at a distance of $35 \mathrm{~km}$ are shown in Fig. 6. It is seen that the performance of PAM4 and QPSK remained largely the same at the two wavelengths (compared to Fig. 5), both in terms of BER and SNR. Extension of the reach from $10 \mathrm{~km}$ to $35 \mathrm{~km}$ introduced some minor performance degradation in both of these formats, which is mainly due to the additional noise arising from the use of the BDFA. Furthermore, although OOK still achieved the lowest BER at the same optical power to the PD amongst the three formats, different degrees of performance degradation were observed in the 1320-nm and 1360-nm cases. In the 1320-nm case, the BER performance of OOK was slightly degraded and the corresponding SNR advantage over QPSK at the same optical power to the PD was only reduced from $\sim 2.5 \mathrm{~dB}$ in the $10-\mathrm{km}$ case to $\sim 2 \mathrm{~dB}$, as shown in Fig. 6(a)\&(b). In contrast, at 1360-nm, a significant performance degradation of the OOK was observed (Fig. 6(c)), which resulted in only a minor PD sensitivity improvement when compared to the QPSK format at the $7 \%$ FEC limit. Accordingly, the SNR advantage of OOK over QPSK was only $\sim 0.5 \mathrm{~dB}$, as shown in Fig. 6(d).

A comparison between the two sets of results at 1320 and $1360 \mathrm{~nm}$ suggests that of the three formats, $\mathrm{OOK}$ has been impacted the most by $\mathrm{CD}$. Performance degradation is not as prominent on PAM4, thanks to its electrical bandwidth being half as broad as that of OOK (therefore, enjoying enhanced tolerance to $\mathrm{CD}$ ). The high tolerance of KK-QPSK to CD-induced power fading is also demonstrated here.
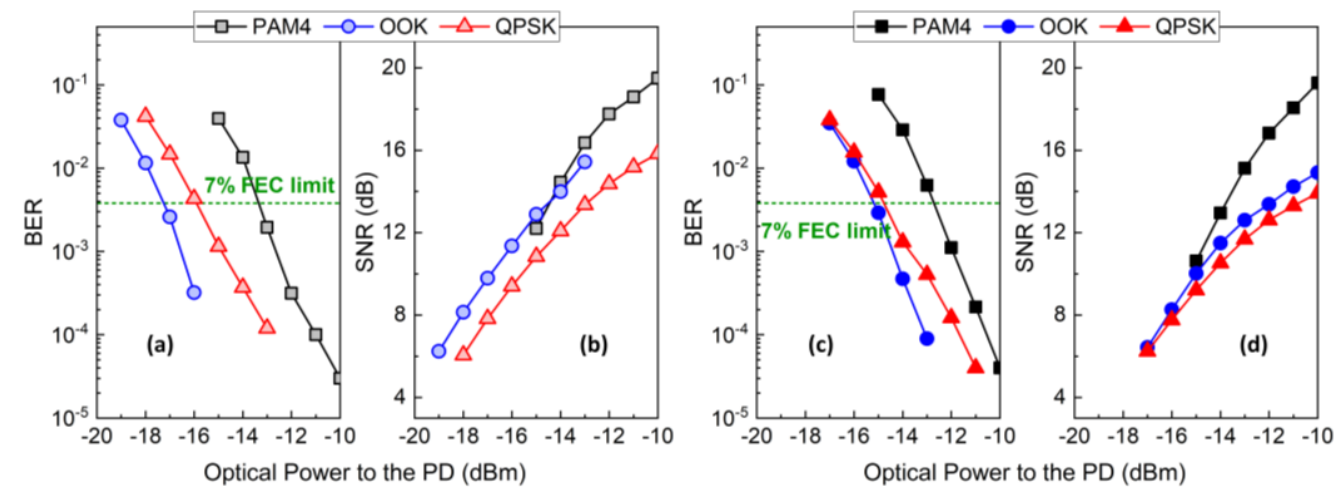

Fig. 6. The transmission performance after 35-km length of SMF at $1320 \mathrm{~nm}$ : (a) BER versus optical power to the PD and (b) SNR versus optical power to the PD; and at $1360 \mathrm{~nm}$ : (c) BER versus optical power to the PD, and (d) SNR versus optical power to the PD. 

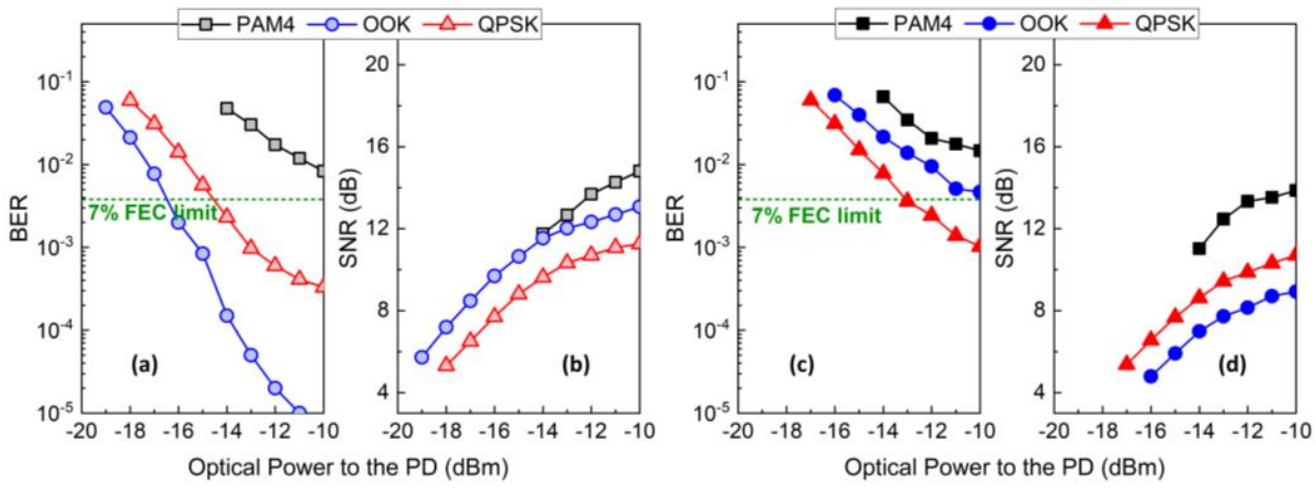

Fig. 7. The transmission performance after 70-km length of SMF at $1320 \mathrm{~nm}$ : (a) BER versus optical power to the PD and (b) SNR versus optical power to the PD; and at $1360 \mathrm{~nm}$ : (c) BER versus optical power to the PD, and (d) SNR versus optical power to the PD.

As mentioned in Section 2, the impact of CD on high-speed IM/DD transmission can be quite significant even within the O-band when the distance is further increased. This is demonstrated through transmission of the three formats over $70 \mathrm{~km}$ of SMF. Figure 7 shows the BER and SNR results of the three formats at $1320 \mathrm{~nm}$ and $1360 \mathrm{~nm}$. For the near zerodispersion wavelength of $1320 \mathrm{~nm}$ shown in Fig. 7(a)\&(b), the 50-Gb/s OOK signal still exhibited more than 1.5-dB optical power sensitivity improvement at the PD compared to 50$\mathrm{Gb} / \mathrm{s}$ QPSK at the 7\% FEC limit. This results from both the CD being too low and thus not having a notable impact on the transmission performance at this data rate and reach (as indicated in Fig. 2), and the recovery of the single-sideband QPSK suffering from a reduced receiver sensitivity to fulfil the KK condition $[17,28]$. Once again, PAM4 exhibited the worst BER performance at any given optical power to the PD; indeed, it was not possible to achieve a BER lower than the 7\% FEC limit with an optical power to the PD of up to $-10 \mathrm{dBm}$ after transmission over this distance. In comparison, as shown in Fig. 7(c)\&(d), at the more dispersive 1360-nm wavelength, the 50-Gb/s QPSK signal which offers enhanced tolerance to $\mathrm{CD}$, achieved the best BER performance amongst the three formats, and was the only one that could support BERs below the 7\% FEC limit. More than 3-dB optical power sensitivity improvement at the PD was obtained compared to OOK. Accordingly, QPSK offered a SNR enhancement of $\sim 2 \mathrm{~dB}$ over OOK, as shown in Fig. 7(d).

\subsection{Comparison of the electrical spectra}

In order to shed some better understanding on the results presented above, we discuss here the features of the electrical spectra of the 50-Gb/s OOK, PAM4 and QPSK signals at both wavelengths after $10-\mathrm{km}$ and $70-\mathrm{km}$ SMF transmission. Fig. 8(a) shows that OOK suffered negligible power fading after $10-\mathrm{km}$ transmission at either wavelength. The power fading effect of OOK was still minor after 70-km transmission at $1320 \mathrm{~nm}$, in contrast to $1360 \mathrm{~nm}$, where severe fading within the signal's bandwidth can be observed at $\sim 17.5 \mathrm{GHz}$, as shown in Fig. 8(b). Note that this agrees well with the simulation result shown in Fig. 2(a). The PAM4 spectra are shown in Figs. 8(c)\&(d) and indicate that power fading was also negligible at both wavelengths after $10-\mathrm{km}$ transmission, as expected. When the reach was extended to $70 \mathrm{~km}$, power fading at $1360 \mathrm{~nm}$ was slightly more severe than that at $1320 \mathrm{~nm}$ due to the higher $C D$. However, in contrast to OOK, no spectral null was experienced within the signal bandwidth, which resulted from the narrower bandwidth of the PAM4 format. In comparison, the singlesideband QPSK modulation ensured that no power fading is present, as shown in Fig. 8(e)\&(f). Note that the DC components of the QPSK signals were excluded in the spectra for ease of presentation. 

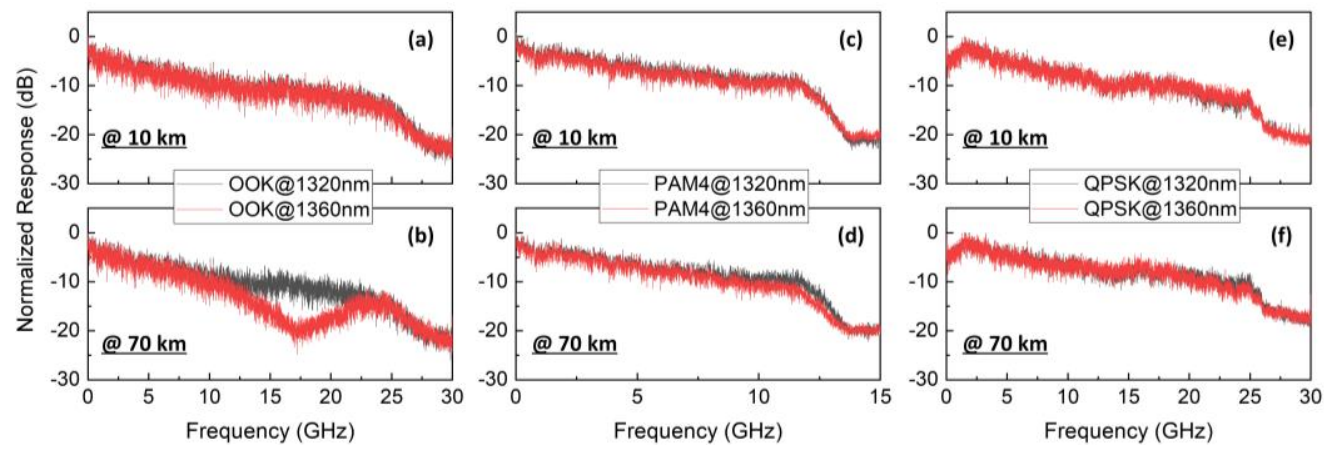

Fig. 8. Comparison of detected signal's electrical spectrum after (a) 10-km transmission using OOK, (b) 70-km transmission using OOK, (c) 10-km transmission using PAM4, (d) 70-km transmission using PAM4, (e) 10-km transmission using QPSK, and (f) 70-km transmission using QPSK.

\subsection{Required optical power to PD versus reach of different formats}

Figure 9 shows the required optical power to the PD versus reach for the three different formats at the BER threshold of $2 \times 10^{-2}$, i.e. the $20 \%$ FEC limit. The results obtained at the two wavelengths of $1320 \mathrm{~nm}$ and $1360 \mathrm{~nm}$ are presented. At both wavelengths, PAM4 suffered the worst performance, as it required the highest optical power to the PD to fulfil the BER requirement. Furthermore, it is shown that for the low-dispersion 1320-nm wavelength, OOK achieved the best PD sensitivity regardless of the transmission distance. In comparison, at the more dispersive 1360-nm wavelength, QPSK exhibited superior PD sensitivity at lengths $>50$ $\mathrm{km}$ where the CD-induced power fading started to impose more significant performance degradation on the OOK transmission.
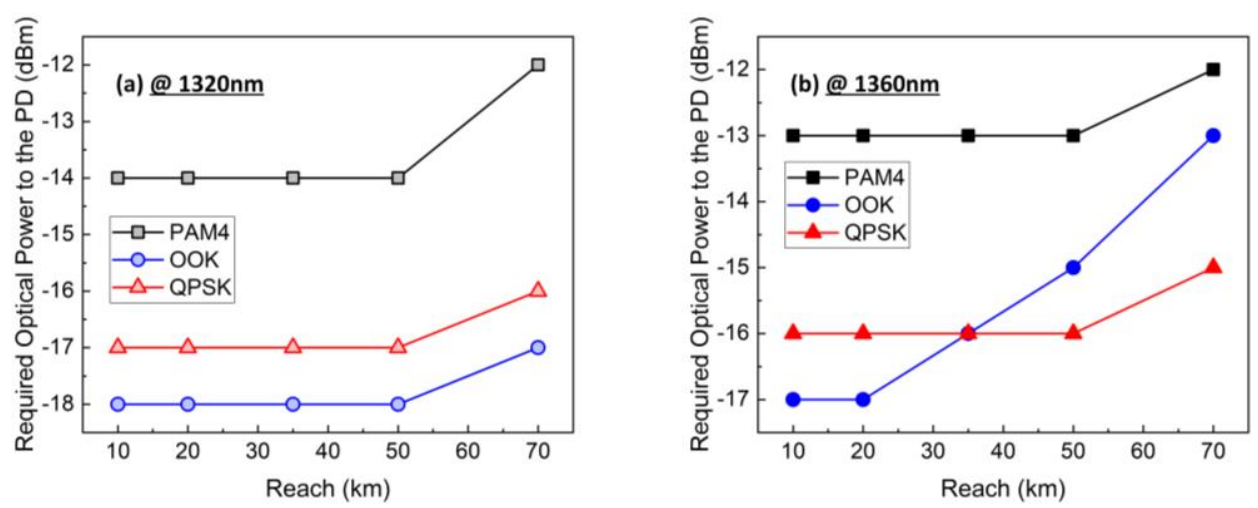

Fig. 9. Required optical power to the PD at the BER threshold of $2 \times 10^{-2}$ for (a) $1320-\mathrm{nm}$ transmission, and (b) 1360-nm transmission.

Table 1 and Table 2 show the BER comparison of the three different formats for the 1320nm and 1360-nm wavelengths, respectively. The optical powers to the PD were fixed to -14 $\mathrm{dBm}$ in all cases. Similar to the receiver sensitivity results shown in Fig. 9, PAM4 suffered the worst performance at both wavelengths. For the low-dispersion 1320-nm wavelength, OOK achieved the lowest BER regardless of the transmission distance. In comparison, at the more dispersive 1360-nm wavelength, QPSK exhibited superior BER performance at lengths $>50$ $\mathrm{km}$ where the CD-induced power fading started to impose significant performance degradation on the OOK transmission.

The results of the two representative O-band wavelengths above indicate that for O-band WDM transmission which can be realized using either frequency combs [29] or multiple laser sources, channels close to the zero-dispersion wavelength will exhibit better performance than 
those at more dispersive O-band wavelengths. It is therefore anticipated that single-sideband modulation (e.g., the KK-QPSK used in this work) will be preferred in longer-reach O-band WDM systems.

Table 1 Comparison of the BERs of different formats after different reaches at $1320 \mathrm{~nm}$

\begin{tabular}{|c|c|c|c|c|c|}
\hline Format & $10 \mathrm{~km}$ & $20 \mathrm{~km}$ & $35 \mathrm{~km}$ & $50 \mathrm{~km}$ & $70 \mathrm{~km}$ \\
\hline PAM4 & $1.1 \times 10^{-2}$ & $1.2 \times 10^{-2}$ & $1.4 \times 10^{-2}$ & $1.5 \times 10^{-2}$ & $4.8 \times 10^{-2}$ \\
\hline OOK & $<1 \times 10^{-4}$ & $<1 \times 10^{-4}$ & $<1 \times 10^{-4}$ & $<1 \times 10^{-4}$ & $1.5 \times 10^{-4}$ \\
\hline QPSK & $2.3 \times 10^{-4}$ & $2.4 \times 10^{-4}$ & $3.7 \times 10^{-4}$ & $4.3 \times 10^{-4}$ & $2.3 \times 10^{-3}$ \\
\hline
\end{tabular}

Table 2 Comparison of the BERs of different formats after different reaches at $1360 \mathrm{~nm}$

\begin{tabular}{cccccc}
\hline Format & \multirow{2}{*}{ Reach } & $20 \mathrm{~km}$ & $35 \mathrm{~km}$ & $50 \mathrm{~km}$ & $70 \mathrm{~km}$ \\
\hline PAM4 & $2.6 \times 10^{-2}$ & $2.7 \times 10^{-2}$ & $2.9 \times 10^{-2}$ & $3.3 \times 10^{-2}$ & $6.6 \times 10^{-2}$ \\
\hline OOK & $<1 \times 10^{-4}$ & $<1 \times 10^{-4}$ & $4.7 \times 10^{-4}$ & $4.8 \times 10^{-3}$ & $2.2 \times 10^{-2}$ \\
\hline QPSK & $6.8 \times 10^{-4}$ & $8 \times 10^{-4}$ & $1.3 \times 10^{-3}$ & $2.7 \times 10^{-3}$ & $7.8 \times 10^{-3}$ \\
\hline
\end{tabular}

\section{Conclusions}

We have presented numerical and experimental studies of the impact of CD on O-band DD transmission. It has been shown that despite the O-band being a low-CD window as a whole, significant performance differences can be experienced between the near-zero-dispersion wavelengths and more dispersive wavelengths. The 1320-nm wavelength can theoretically support 5.4 times longer reach than that of the 1360-nm wavelength thanks to its lower CD. We have further experimentally evaluated the impact of $\mathrm{CD}$ on the transmission performance of 50-Gb/s PAM4, OOK and single-sideband QPSK formats in the O-band at distances that extended to $70 \mathrm{~km}$ using a BDFA as a pre-amplifier. The experimental results show that although OOK achieves the best receiver sensitivity at wavelengths where the dispersion is near-zero, in more dispersive regions of the O-band, the QPSK format becomes preferable, highlighting that over such distances, the $\mathrm{CD}$ at longer wavelengths of the $\mathrm{O}$-band transmission can no longer be neglected. In these instances, single-sideband QPSK offers the best performance: it is demonstrated that more than 3-dB receiver sensitivity improvement over OOK can be achieved after 70-km SMF transmission at $1360 \mathrm{~nm}$. We believe that our results offer an insight into the possible evolution of O-band transmission towards longer-distance, higher-capacity WDM transmission, which can be enabled by novel fiber amplification technologies.

\section{Funding}

Engineering and Physical Sciences Research Council (EPSRC) Airguide Photonics Programme Grant (EP/P030181/1) and EPSRC PHOS project (EP/S002871/1).

\section{Acknowledgments}

The data for this work is accessible through the University of Southampton Institutional Research Repository (DOI:10.5258/SOTON/xxxxx).

\section{Disclosures}

The authors declare no conflicts of interest.

\section{References}

1. E. Agrell, M. Karlsson, A. R. Chraplyvy, D. J. Richardson, P. M. Krummrich, P. Winzer, K. Roberts, J. K. Fischer, S. J. Savory, B. J. Eggleton, M. Secondini, F. R. Kschischang, A. Lord, J. Prat, I. Tomkos, J. E. Bowers, S. Srinivasan, M. Brandt-Pearce, and N. Gisin, "Roadmap of optical communications," J. Opt., 18, 1-40 (2016).

2. N. Sambo, A. Ferrari, A. Napoli, N. Costa, J. Pedro, B. Sommerkorn-Krombholz, P. Castoldi, and V. Curri, "Provisioning in Multi-band Optical Networks," J. Light. Technol., 38(9), 2598-2605 (2020). 
3. T. Hayashi, T. Nakanishi, K. Hirashima, O. Shimakawa, F. Sato, K. Koyama, A. Furuya, Y. Murakami, and T. Sasaki, "125- $\mu \mathrm{m}$-cladding eight-core multi-core fiber realizing ultra-high-density cable suitable for O-Band short-reach optical interconnects," J. Light. Technol., 34(1), 85-92 (2016).

4. X. Pang, O. Ozolins, L. Zhang, A. Udalcovs, R. Lin, R. Schatz, U. Westergren, S. Xiao, W. Hu, G. Jacobsen, S. Popov, and J. Chen, "Beyond $200 \mathrm{Gbps}$ per lane intensity modulation direct detection (IM/DD) transmissions for optical interconnects: challenges and recent developments," in Proc. of OFC, paper W4I.7 (2019).

5. G. Lu, H. Zhang, S. Shinada, J. Hong, Y. Cheng, and S. Yokoyama, "Power-efficient O-band 40 Gbit/s PAM4 transmitter based on dual-drive cascaded carrier-depletion and carrier-injection silicon Mach-Zehnder modulator with binary driving electronics at CMOS voltages" IEEE J. Sel. Top. Quantum Electron., 27(3), 3500308 (2021).

6. L. Chorchos, and J.P. Turkiewicz, "O-Band $8 \times 100 \mathrm{G}$ data transmission with $240 \mathrm{GHz}$ channel spacing," IEEE Photon. Technol. Lett., 23(12), 2288-2291 (2019).

7. M. Moralis-Pegios, S. Pitris, T. Alexoudi, N. Terzenidis, H. Ramon, J. Lambrecht, J. Bauwelinck, X. Yin, Y. Ban, P. Heyn, J. Campenhout, T. Lamprecht, A. Lehnman, and N. Pleros, "4-channel $200 \mathrm{~Gb} / \mathrm{s}$ WDM O-band silicon photonic transceiver sub-assembly," Opt. Express, 28(4), 5706-5714 (2020).

8. Chris Cole, "1310 nm vs 1550 nm," presented at ECOC 2017. [Online]. Available: https://www.finisar.com/sites/default/files/resources/1310nm_vs_1550nm_ecoc2017.pdf.

9. V. Mikhailov, M.A. Melkumov, D. Inniss, A. M. Khegai, K. E. Riumkin, S. V. Firstov, F. V. Afanasiev, M. F. Yan, Y. Sun, J. Luo, G. S. Puc, S. D. Shenk, R. S. Windeler, P. S. Westbrook, R. L. Lingle, E. M. Dianov, and D. J. DiGiovanni, "Simple broadband bismuth doped fiber amplifier (BDFA) to extend O-band transmission reach and capacity," in Proc. of OFC, paper M1J.4 (2019).

10. E.M. Dianov., "Amplification in extended transmission bands using bismuth-doped optical fibers," J. Light. Technol., 31(4), 681-688 (2013).

11. M.A. Melkumov, V. Mikhailov, A.M. Khegai, K. E. Riumkin, S. V. Firstov, F. V. Afanasiev, A. N. Guryanov, M. F. Yan, Y. Sun, J. Luo, G. S. Puc, S. D. Shenk, R. S. Windeler, P. S. Westbrook, R. L. Lingle, D. J. DiGiovanni and E. M. Dianov, " 25 Gbs-1 data transmission using a bismuth-doped fibre amplifier with a gain peak shifted to $1300 \mathrm{~nm}, "$ Quantum Electron., 48(11), 989-992 (2018).

12. N. K. Thipparapu, A. A. Umnikov, P. Barua, and J. K. Sahu, "Bi-doped fiber amplifier with a flat gain of $25 \mathrm{~dB}$ operating in the wavelength band 1320-1360 nm," Opt. Lett., 41(7), 1518-1521 (2016).

13. Y. Wang, N. K. Thipparapu, D. J. Richardson, and J. K. Sahu, "Broadband bismuth-doped fiber amplifier with a record 115-nm bandwidth in the O and E bands," in Proc. of OFC, paper Th4B.1 (2020).

14. N. Taengnoi, K.R.H. Bottrill, N.K. Thipparapu, A. A. Umnikov, J. K. Sahu, P. Petropoulos, and D. J. Richardson, "WDM transmission with in-line amplification at $1.3 \mu \mathrm{m}$ using a Bi-doped fiber amplifier," J. Light. Technol., 37(8), 1826-1830 (2019).

15. N. Taengnoi, K. R. H. Bottrill, Y. Hong, N. K. Thipparapu, C. Lacava, J. K. Sahu, D. J. Richardson, and P. Petropoulos, "Reach extension of PAM4 signals in O-band transmission by application of alternate-markinversion," in Proc. of ECOC, paper P93 (2019).

16. Y. Hong, H. Sakr, N. Taengnoi, K. R. H. Bottrill, T. D. Bradley, J. R. Hayes, G. T. Jasion, H. Kim, N. K. Thipparapu, Y. Wang, A. A. Umnikov, J. K. Sahu, F. Poletti, P. Petropoulos, and D. J. Richardson, "Multi-band direct-detection transmission over an ultrawide bandwidth hollow-core NANF," J. Light. Technol., 38(10), 28492857 (2020).

17. Y. Hong, K. R. H. Bottrill, N. Taengnoi, N. K. Thipparapu, Y. Wang, A. A. Umnikov, J. K. Sahu, D. J. Richardson, and P. Petropoulos, "Experimental demonstration of dual O+C-band WDM transmission over 50km SSMF with direct detection," J. Light. Technol., 38(8), 2278-2284 (2020).

18. Y. Hong, K. R. H. Bottrill, N. Taengnoi, N. K. Thipparapu, Y. Wang, J. K. Sahu, D. J. Richardson, and P. Petropoulos, "First investigation on double- and single-sideband formats in BDFA-enabled O-band transmission," in Proc. of CLEO, paper STh3L.3 (2020).

19. IEEE P802.3bs $200 \mathrm{~Gb} / \mathrm{s}$ and $400 \mathrm{~Gb} / \mathrm{s}$ Ethernet Task Force. [Online]. Available: http://grouper.ieee.org/groups/802/3/bs/.

20. M. S. Erkılınç, S. Kilmurray, R. Maher, M. Paskov, R. Bouziane, S. Pachnicke, H. Griesser, B. C. Thomsen, P. Bayvel, and R. I. Killey, "Nyquist-shaped dispersion-precompensated subcarrier modulation with direct detection for spectrally-efficient WDM transmission," Opt. Express, 22(8), 9420-9431 (2014).

21. X. Chen, C. Antonelli, A. Mecozzi, D. Che, and W. Shieh, "High-capacity direct-detection systems", Optical Fiber Telecommunications VII, Chapter 10, Academic Press (2020).

22. J. Zhou, H. Wang, Y. Feng, W. Liu, S. Gao, C. Yu, and Z. Li, "Processing for dispersive intensity-modulation and direct-detection fiber-optic communications," Opt. Lett., 46(1), 138-141 (2021).

23. M. Amemiya, "Pulse broadening due to higher order dispersion and its transmission limit," J. Light. Technol., 20(4), 591-597 (2002).

24. N. Taengnoi, K. R. H., Bottrill, Y. Hong, Y. Wang, N. K. Thipparapu, J. K. Sahu, P. Petropoulos, and D. J. Richardson, "Experimental Characterization of Bismuth-Doped Fibre Amplifier: Electrical NF, PDG, and XGM," in Proc. of CLEO, paper SW3R.2 (2020).

25. H. Sakr, Y. Hong, T. D. Bradley, G. T. Jasion, J. R. Hayes, H. Kim, I. A. Davidson, E. N. Fokoua, Y. Chen, K. R. H. Bottrill, N. Taengnoi, N. V. Wheeler, P. Petropoulos, D. J. Richardson, and F. Poletti, "Interband short reach data transmission in ultrawide bandwidth hollow core fiber," J. Light. Technol., 38(1), 159-165 (2020).

26. A. Mecozzi, C. Antonelli, and M. Shtaif, "Kramers-Kronig coherent receiver," Optica, 3(11), 1220-1227 (2016). 
27. B. Sun, D. Che, H. Ji, and W. Shieh, "Towards low carrier-to-signal power ratio for Kramers-Kronig receiver" in Proc. of OFC, paper M1H.6 (2019).

28. T. Bo, and H. Kim, "Kramers-Kronig receiver operable without digital upsampling," Opt. Express, 26(11), 13810-13818 (2018).

29. H. Hu, and L. K. Oxenløwe, "Chip-based optical frequency combs for high-capacity optical communications," Nanophotonics, 10(5), 1367-1385 (2021). 